Supporting Information

\title{
Rational Design of a Humanized Antibody Inhibitor of Cathepsin B
}

\author{
Zhefu Dai ${ }^{1}$, Qinqin Cheng ${ }^{1}$ and Yong Zhang ${ }^{1,2,3,4^{*}}$
}

${ }^{1}$ Department of Pharmacology and Pharmaceutical Sciences, School of Pharmacy, University of Southern California, Los Angeles, CA 90089

${ }^{2}$ Department of Chemistry, Dornsife College of Letters, Arts and Sciences, University of Southern California, Los Angeles, CA 90089

${ }^{3}$ Norris Comprehensive Cancer Center, University of Southern California, Los Angeles, CA 90089

${ }^{4}$ Research Center for Liver Diseases, University of Southern California, Los Angeles, CA 90089

*Email: yongz@usc.edu 

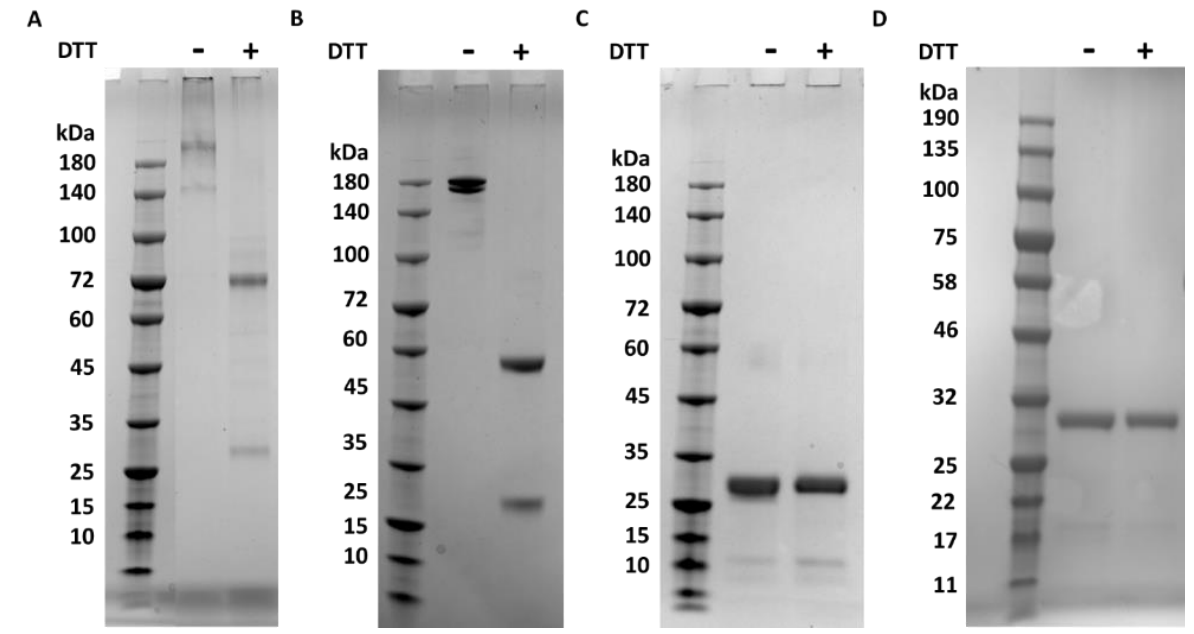

Figure S1. SDS-PAGE gels of purified proteins. (A) CTSB IgG. (B) Herceptin. (C) Human CTSB. (D) Human CTSB C29A mutant. 


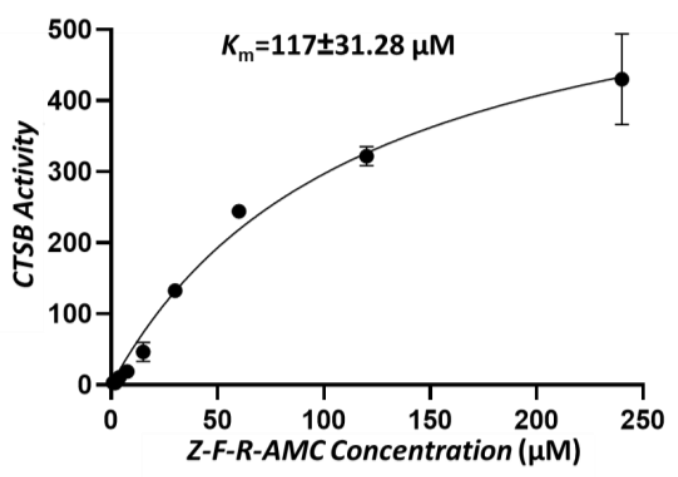

Figure S2. Enzymatic parameter of the recombinant human CTSB proteolytic activity with Z-PheArg-AMC (Z-F-R-AMC) as a substrate. 